\title{
Pulmonary veno-occlusive disease in association with Hodgkin's disease
}

\author{
SJ CAPEWELL, AJ WRIGHT, DA ELLIS \\ From the Cardiothoracic Centre, Freeman Hospital, Newcastle upon Tyne
}

Pulmonary veno-occlusive disease is a rare cause of pulmonary hypertension of unknown aetiology. Pulmonary veno-occlusive disease is usually an isolated condition but has been reported in association with autoimmune vasculitis ${ }^{1}$ and may follow viral or toxoplasmal infection. ${ }^{2}$ We report a case of pulmonary veno-occlusive disease in association with Hodgkin's disease, a combination not previously described, and consider what treatment could be used to lower the pulmonary arterial pressure.

\section{Case report}

A 22 year old man, a gardener, who had previously been well, presented with a four month history of increasing dyspnoea, non-productive cough, retrosternal chest discomfort, and weight loss. On examination he had extensive acne vulgaris and bilateral axillary lymphadenopathy. The extremities were cool but no other abnormal physical signs were found. A biopsy specimen of a lymph node showed appearances of reactive hyperplasia. When reviewed four weeks later he was unable to walk more than about 45 metres on level ground because of breathlessness. He had sinus tachycardia, raised venous pressure, accentuation of the right ventricular impulse, a delayed and loud pulmonary second heart sound, and a third heart sound audible at the left sternal edge. No abnormal signs were found in the lungs.

The chest radiograph showed large proximal pulmonary arteries, widespread linear opacities and scattered micronodular opacities throughout the lung fields, and a small right pleural effusion. The electrocardiogram and the two dimensional echocardiogram showed right ventricular hypertrophy. The results of routine haematological, biochemical, and clotting studies and serum immunoglobulin and complement concentrations were normal. No evidence of recent viral or toxoplasmal infection was found. Tuberculin and Kveim test reactions were negative. Transbronchial lung biopsy specimens showed appearances of non-specific cellular infiltration of alveolar walls. An isotope lung scan showed normal ventilation but grossly disturbed perfusion suggestive of multiple pulmonary emboli. Right heart catheterisation confirmed the presence of severe pulmonary hypertension $(86 / 29 \mathrm{~mm}$ $\mathrm{Hg}$ ) and the mean pulmonary wedge pressure was recorded, with difficulty, as $31 \mathrm{~mm} \mathrm{Hg}$. Pulmonary angio-

Address for correspondence (reprints will not be available): Dr DA Ellis, District General Hospital, Lowestoft Road, Gorleston, Great Yarmouth.

Accepted 1 February 1984 graphy showed enlarged proximal pulmonary arteries with $\overrightarrow{\vec{\omega}}$ no intra-arterial filling defects. Most of the flow was diverted to large vessels running through the medial parts of both lower lobes. In other areas considerable narrowing of tertiary and quarternary branches was seen.

A gallium-67 scan was performed, which showed low $i v$ activity over the lungs. Activity was concentrated in the left of axilla and there was no increase in activity over the mediastinum. A left axillary lymph node biopsy specimen showed histological features of nodular sclerosing Hodg- kin's disease (fig 1). Lymphangiography, liver biopsy and $\subseteq$ bone marrow biopsy yielded normal results; a staging laparotomy was not performed.

The possibility of pulmonary lesions caused by lymphoma was considered and two courses of chemotherapy with mustine, vincristine, procarbazine, and prednisolone (MOPP) were given. No improvement occurred. Because of the atypical radiographic features, severe pulmonary hypertension, and the absence of lymphoma elsewhere an open lung biopsy was then performed. Sections of the lung taken from the middle lobe showed the features of pulmonary veno-occlusive disease. A large pulmonary vein showed extensive intimal proliferation incorporating elastic tissue and muscle (fig 2). Smaller vessels showed considerable intimal proliferation with collagen extending into the pulmonary venules. Alveolar capillary walls were thickened and haemosiderin laden macrophages were present in the alveolar spaces (fig 3). Pulmonary arterioles showed the features of pulmonary hypertension without plexiform

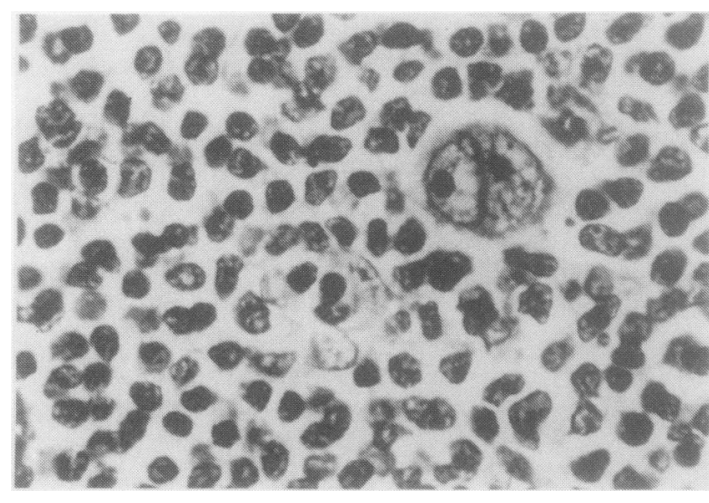

Fig 1 Binucleated Sternberg Reed cell in a lymph node. (Haematoxylin and eosin, $\times 695$.) 


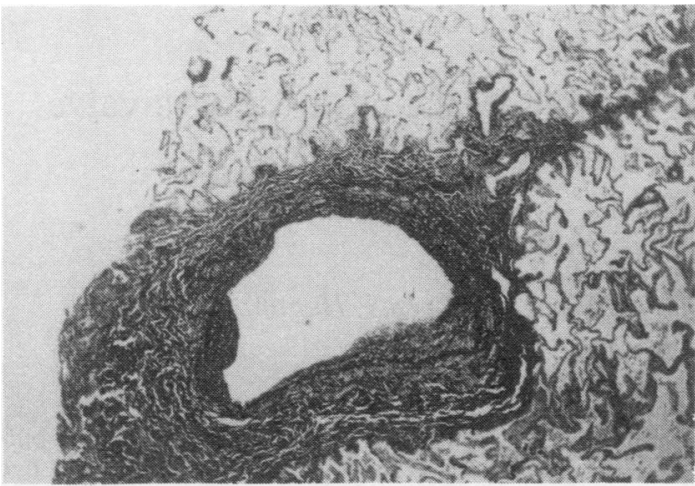

Fig 2 Pulmonary vein in an interlobular septum showing irregular fibroelastic intimal thickening. (Elastic van giesen, $\times$ 30.)

lesions or necrotising arteritis. There was no evidence of Hodgkin's disease in the lung. Because of severe breathlessness empirical treatment was given with prednisolone $60 \mathrm{mg}$ daily and azathioprine $150 \mathrm{mg}$ daily pending the lung biopsy report. Anticoagulation with warfarin was started because of the risk of in situ thrombosis and the perfusion lung scan appearance. No improvement was seen after six weeks and the response to vasodilator agents and oxygen was studied. Pulmonary artery pressure with the patient breathing air was 91/41 (mean 65) $\mathrm{mm} \mathrm{Hg}$ and while he was breathing $60 \%$ oxygen it fell to $66 / 31$ (mean 43) $\mathrm{mm} \mathrm{Hg}$. After an isosorbide infusion $(50 \mu \mathrm{g} / \mathrm{min})$ it was $52 / 37$ (mean 46 ) $\mathrm{mm} \mathrm{Hg}$ and after oral hydrallazine $150 \mathrm{mg}$ per day for five days it was $47 / 22$ (mean 31) mm Hg. Breathing $60 \%$ oxygen while he was receiving hydrallazine lowered the pulmonary artery pressure to $33 / 17$ (mean 23) $\mathrm{mm} \mathrm{Hg}$.

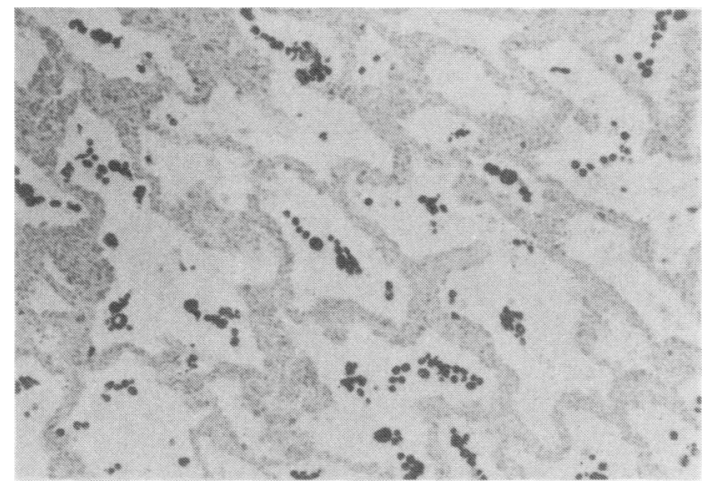

Fig 3 Haemosiderin laden macrophages within thick walled alveoli. (Perls' stain, $\times 78$.)
Prednisolone was withdrawn and treatment with hydrallazine, oxygen, azathioprine, and warfarin continued. Symptomatic improvement occurred over the next four weeks, with an increase in exercise tolerance to about 100 $\mathrm{m}$ on level ground The patient was discharged from hospital and for four months there was no clinical deterioration, although symptoms were variable from day to day. He died suddenly while away from home on holiday five months after starting treatment with hydrallazine and 12 months after the onset of symptoms. No necropsy was performed.

\section{Discussion}

Pulmonary veno-occlusive disease is a rare condition with a poor prognosis, most patients surviving less that two years from diagnosis. ${ }^{3}$ The diagnosis may be suspected when the clinical signs of pulmonary hypertension are accompanied by radiological signs of pulmonary oedema in the absence of structural heart disease $e^{4}$ but is usually made by histological examination of lung tissue obtained either at open lung biopsy or at necropsy. Treatment has been disappointing; corticosteroids have failed to produce improvement ${ }^{3}$ and vasodilators used briefly in one patient gave unconvincing results. ${ }^{5}$ The reported exception was a 46 year old woman with pulmonary veno-occlusive disease and autoimmune vasculitis who responded to azathioprine and was alive four years later.' In our patient hydrallazine produced a sustained fall in pulmonary arterial pressure that was enhanced by breathing $60 \%$ oxygen and we think that this was largely responsible for the observed clinical improvement, although prognosis was probably not affected. The association with Hodgkin's disease remains unexplained.

We are grateful to Professor D Heath for his report on the lung biopsy specimen, to Dr T Ashcroft for the photomicrographs, and to Dr S Nariman for permission to report the case.

\section{References}

' Sanderson JE, Spiro SG, Hendry AT, Turner-Warwick M. A case of pulmonary veno-occlusive disease responding to treatment with azathioprine. Thorax 1977;32:140-8.

${ }^{2}$ Anonymous. Pulmonary veno-occlusive disease. $\mathrm{Br} \mathrm{Med} \mathrm{J}$ 1972; iii: 369.

${ }^{3}$ Thadani U, Burrow D, Whitaker W, Heath D. Pulmonary veno-occlusive disease. $Q J$ Med 1975; 173:133-59.

${ }^{4}$ Brown $\mathrm{CH}$, Harrison CV. Pulmonary veno-occlusive disease. Lancet 1966;ii:61-6.

${ }^{5}$ Rosenthal A, Wawter G, Wagenvoort CA. Intrapulmonary veno-occlusive disease. Am J Cardiol 1973;31:78-81. 